\title{
FEM/wideband FMBEM coupling to model fluid-structure interaction
}

\author{
L. L. Chen, C. J. Zheng \& H. B. Chen \\ Department of Modern Mechanics, CAS Key Laboratory of Mechanical \\ Behavior and Design of Materials, University of Science and Technology \\ of China, P. R. China
}

\begin{abstract}
A coupling algorithm based on the finite element method and the wideband fast multipole boundary element method (FEM/wideband FMBEM) is proposed for the simulation of fluid-structure interaction and structural acoustic sensitivity analysis using the direct differentiation method. The iterative solver GMRES is applied to accelerate the solution of the linear system of equations. The FEM/Wideband FMBEM algorithm makes it possible to predict the effects of arbitrarily shaped vibrating structures on the sound field numerically.

Keywords: fluid-structure interaction, FEM, wideband FMBEM, design sensitivity analysis, direct differentiation method.
\end{abstract}

\section{Introduction}

A suitable approach for fluid-structure interaction problems and the associated predictions is the coupled FEM/BEM [1,2]. But the coupling analysis of structuralacoustic underwater based on FEM/Conventional BEM (CBEM) algorithm still represents the bottleneck of large computation cost, because the CBEM produces a dense and non-symmetrical coefficient matrix which induces $O\left(N^{3}\right)$ arithmetic operations to solve the system of equations directly, such as by using the Gauss elimination method. The fast multipole method (FMM) [3,4] allows the matrixvector product to be performed to a given precision in $O(N)$ operations and reduces the storage requirements to $O(N)$ as well, for instance, for potential problems and low-frequency acoustic wave problems.

Acoustic design sensitivity analysis can provide information on how the geometry change affects the acoustic performance of the given structure, so it 
is an important step of the acoustic design and optimization processes [5, 6]. But the sensitivity analysis of the structural-acoustic based on FEM/Conventional BEM algorithm represents the bottleneck in computation efforts. In this paper, the coupling algorithm FEM/Wideband FMBEM is applied to the structural-acoustic sensitivity analysis using direct differentiation method.

This work promotes the applications of coupling FEM/Wideband FMBEM in the fluid-structure interaction problems. The example of scattering from elastic spherical shell underwater is presented to demonstrate the accuracy and efficiency of this method.

\section{Structural-acoustic analysis}

\subsection{FEM modeling}

It is assumed that a harmonic load with the excitation frequency $\omega$ is applied to the structure, the steady-state response of the structure can be calculated from the frequency-response analysis. The linear system of equations to compute the nodal displacements $u$ is derived by

$$
\left(\mathbf{K}+i \omega \mathbf{C}-\omega^{2} \mathbf{M}\right) \mathbf{u}(\omega)=\mathbf{A} \mathbf{u}=\mathbf{f},
$$

where $i=\sqrt{-1}, \mathbf{M}$ the mass matrix, $\mathbf{K}$ the stiffness matrix, $\mathbf{C}$ the damping matrix and $\mathbf{u}$ the nodal displacement vector. Note that the steady-state response has the same frequency as the applied load but may have a different phase angle due to the existence of damping. If the applied load is not harmonic, Eq. (1) can still be applied by decomposing the time-dependent forces into the frequency domain. Taking into account the effect of the acoustic pressure at the structural surfaces, we apply an acoustic load $\mathbf{C}_{\text {sf }} \mathbf{p}$ along with the structural load $\mathbf{f}_{\mathbf{s}}$, and then the excitation can be expressed as:

$$
\mathbf{f}=\mathbf{f}_{\mathrm{s}}+\mathbf{C}_{\mathrm{sf}} \mathbf{p}
$$

where the coupling matrix $\mathbf{C}_{\mathbf{s f}}$ transforms the degrees of freedom of the fluid to the structural degrees of freedom, and it can be expressed as:

$$
\mathbf{C}_{\mathbf{s f}}=\int_{\Gamma_{\text {int }}} \mathbf{N}_{\mathbf{s}}^{\mathbf{T}} \mathbf{n} \mathbf{N}_{\mathbf{f}} d \Gamma_{i n t}
$$

where $\Gamma_{\text {int }}$ denotes the interaction surface, $\mathbf{N}_{\mathbf{s}}$ and $\mathbf{N}_{\mathbf{f}}$ are the global interpolation functions for the structure and fluid domains, respectively, $\mathbf{n}$ is the surface normal vector. By substituting Eq. (2) into Eq. (1), we can obtain the following formula

$$
\mathbf{u}=\mathbf{A}^{-1} \mathbf{f}_{\mathbf{s}}+\mathbf{A}^{-1} \mathbf{C}_{\mathbf{s f}} \mathbf{p}
$$




\subsection{BEM modeling}

The Helmholtz equation can be reformulated into a boundary integral equation (BIE) defined on the structure boundary $\Gamma$ as follows:

$$
c(x) p(x)+\int_{\Gamma} q^{*}(x, y) p(y) \mathrm{d} \Gamma(y)=\int_{\Gamma} p^{*}(x, y) q(y) \mathrm{d} \Gamma(y)+p_{i}(x) .
$$

If the boundary $\Gamma$ is divided into $N$ elements (e.g. using piecewise constant discretization in this study). Then, after collecting the equations for all collocation points (nodes) located at the centre of each element and expressing them in matrix forms, one can obtain the following system of linear algebraic equations

$$
\mathbf{H p}=\mathbf{G q}+\mathbf{p}_{\mathbf{i}}
$$

\subsection{FEM/BEM modeling}

The governing equations shown above are linked up via the continuity condition $\mathbf{q}=-i \omega \rho \mathbf{v}$ across the interaction surface. The normal velocity $\mathbf{v}$ can be expressed as a function of the displacement $\mathbf{u}$, as follows

$$
\mathbf{v}=i \omega \mathbf{S}^{-1} \mathbf{C}_{\mathbf{f s}} \mathbf{u}
$$

By substituting Eq. (7) into Eq. (6), we can obtain the following formulation

$$
\mathbf{H p}=\omega^{2} \rho \mathbf{G S} \mathbf{S}^{-\mathbf{1}} \mathbf{C}_{\mathbf{f s}} \mathbf{u}+\mathbf{p}_{\mathbf{i}}
$$

By substituting Eq. (4) into Eq. (8), one can obtain the following coupled boundary element equation

$$
\mathbf{H p}-\mathbf{G W C}_{\mathbf{s f}} \mathbf{p}=\mathbf{G W f}_{\mathrm{s}}+\mathbf{p}_{\mathbf{i}} .
$$

Fast multipole method(FMM) and the iterative solver GMRES were applied to accelerate the solution of the coupled boundary element system equation, and it will need $O(N)$ operations to solve the coupled boundary element system equation. In this paper we will use a sparse direct solver to solve the symmetric and frequency-dependent system of linear equation $\mathbf{A x}=\mathbf{y}$.

\subsection{Shape design sensitivity analysis for coupled boundary element equation}

First, by differentiating the coupled boundary element equation (9) with respect to the shape design variable, we can obtain the following formulation

$$
\mathbf{H} \dot{\mathbf{p}}-\mathbf{G W C}_{\mathrm{sf}} \dot{\mathbf{p}}=\dot{\mathbf{G}} \mathbf{a}+\mathbf{G b}-\dot{\mathbf{H}} \mathbf{p}+\dot{\mathbf{p}}_{\mathbf{i}}
$$

where vectors $\mathbf{a}$ and $\mathbf{b}$ are defined by

$$
\mathbf{a}=\mathbf{W C _ { \mathbf { s f } }} \mathbf{p}+\mathbf{W} \mathbf{f}_{\mathbf{s}},
$$




$$
\begin{gathered}
\mathbf{b}=\dot{\mathbf{W}} \mathbf{C}_{\mathbf{s f}} \mathbf{p}+\mathbf{W} \dot{\mathbf{C}}_{\mathbf{s f}} \mathbf{p}+\dot{\mathbf{W}} \mathbf{f}_{\mathbf{s}}+\mathbf{W} \dot{f}_{\mathbf{s}}, \\
\dot{\mathbf{W}}=\omega^{2} \rho\left(\mathbf{S}^{-1} \mathbf{C}_{\mathbf{f s}} \mathbf{A}^{-1}+\mathbf{S}^{-1} \dot{\mathbf{C}}_{\mathbf{f s}} \mathbf{A}^{-1}+\mathbf{S}^{-1} \mathbf{C}_{\mathbf{f s}} \dot{A}^{-1}\right) .
\end{gathered}
$$

After obtaining all the unknown boundary acoustic pressure values by solving Eq. (9) and subsequently substituting all the boundary acoustic pressure into Eq. (10), we can get the computational solution of the matrix-vector products on the right hand side of Eq. (10). In fact, the expressions of matrices determining vector $\mathbf{b}$, such as $\dot{\mathbf{S}}^{-\mathbf{1}}, \dot{\mathbf{C}}_{\mathbf{f s}}$ and $\dot{\mathbf{C}}_{\mathbf{s f}}$, can be complicated especially when the structural domain is approximated using shell finite elements. And so it is very difficult to solve them directly. But the semi-analytical derivative method can be applied to conquer the difficulty.

It is worth noting that solving directly the inverse of matrix $\mathbf{A}$ in Eq. (13) will be very expensive and it is very difficult to get the variation of inverse of matrix A by using directly the finite difference method. But $\mathbf{A}^{-1}$ can be replaced by the following formulation

$$
\dot{\mathbf{A}}^{-1}=\mathbf{A}^{-1} \dot{\mathbf{A}} \mathbf{A}^{-1}
$$

By substituting Eq. (14) into Eq. (13), we can obtain efficiently the solution of $\dot{\mathbf{W}} \mathbf{y}$ by solving directly $\mathbf{A x}=\mathbf{y}$. In fact, it needs much computing time to solve directly matrices $\mathbf{H}, \mathbf{G}, \dot{\mathbf{H}}$ and $\dot{\mathbf{G}}$ in Eq. (10) by using conventional BEM since the matrices are full and un-symmetric. But, fast multipole method and the iterative solver GMRES can be applied to accelerate the matrix-vector products.

\section{The wideband FMM formulations}

In this section, the FMM approach is introduced to accelerate the matrix-vector product in Eqs. (9) and (10) and the iterative solver GMRES is used to solve the coupled boundary element equation and its sensitivity equation. It is well-known that the original FMM is inefficient for high frequency problems, so the diagonal form (high-frequency method) is introduced to overcome this difficulty. But the diagonal form has instability problem for the solution of low frequency Helmholtz equations. The wideband FMM obtained by combining the original FMM and the diagonal form FMM can overcome the above problems. In the wideband FMM, we use the following M2F formula to convert the moment of the original FMM to that of the diagonal FMM [4]:

$$
F(k, \hat{\mathbf{k}}, O)=\sum_{n=0}^{\infty} \sum_{m=-n}^{n}(2 n+1) \mathrm{i}^{-n} Y_{n}^{m}(\hat{\mathbf{k}}) M_{n}^{m}(k, O) .
$$

The local expansion coefficient of the diagonal FMM can also be converted to that of the original FMM by using the following H2L formula:

$$
L_{n}^{m}\left(k, x^{\prime}\right)=\frac{\mathrm{i}^{n}}{4 \pi} \int_{S} Y_{n}^{m}(\hat{\mathbf{k}}) H\left(k, \hat{\mathbf{k}}, x^{\prime}\right) \mathrm{d} S .
$$


Actually, the number of terms used in the functions $O, I, M$ and $L$ and the number of the plane wave samples $\hat{\mathbf{k}}$ along the unit sphere have to be truncated. The number of truncation terms and the plane wave samples depends on the size $d$ of the cell and the wave number $k$. It is given in the following form in [7]:

$$
p=k d+c \cdot \log (k d+\pi) .
$$

where $c$ is a constant. Obviously, a larger $c$ relates to a larger truncation number $p$ and it normally leads to an improvement of accuracy but induces to a longer computing time and larger memory usage. Thus, it is a key parameter in the FMM algorithm, which is chosen as 5 in this paper [6].

\subsection{Scattering from an elastic spherical shell}

In this example, we consider the acoustic scattering of a plane incident wave with unit amplitude on an spherical shell with radius $a=5.0 m$ centred at point $(0,0,0)$, and the plane incident wave is travelling along the positive $\mathbf{x}$ axis, as shown in Fig. 1. For the spherical shell, the thickness is chosen as $0.15 \mathrm{~m}$, Young's modulus $2.07 \times 10^{11} \mathrm{~Pa}$, Poisson's ratio 0.3 and the density $7669 \mathrm{~kg} / \mathrm{m}^{3}$. For the fluid, the density is chosen as $1000 \mathrm{~kg} / \mathrm{m}^{3}$ and the speed of sound $1524 \mathrm{~m} / \mathrm{s}$. The analytical solution to which the numerical results will be compared is the series solution published by Junger and Feit [8].

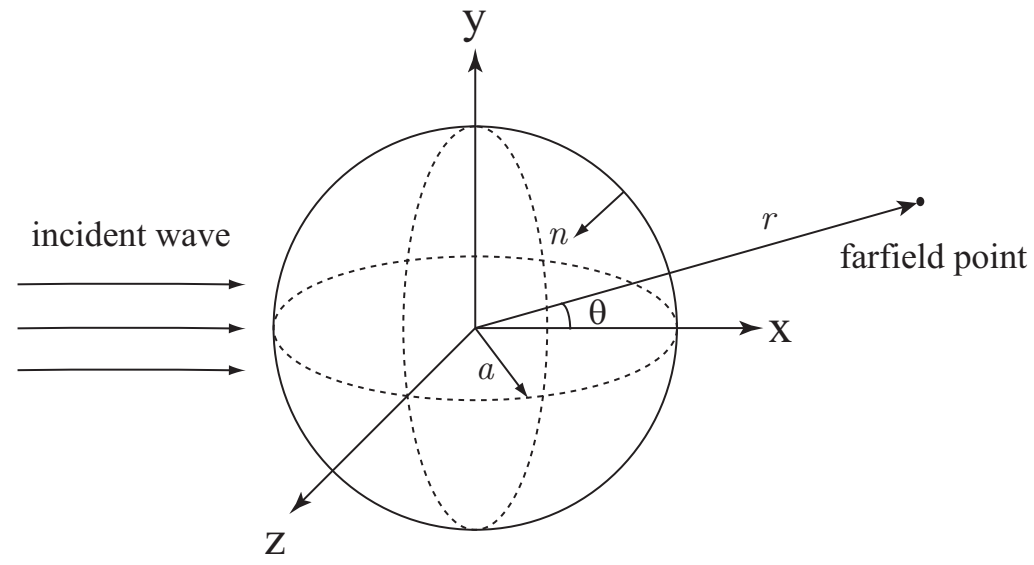

Figure 1: Scattering from an spherical shell with radius $a$.

The shell was modeled with first order triangular shell element. Figure 2 shows a low-frequency comparison between the coupling FEM/Wideband FMBEM calculation and the series solution for the scattered pressure on the farfield points distributed on $\mathbf{x y}$ plane. The ordinate of this figure is the normalized pressure $p_{1}=\left|p r / p_{o} a\right|$, where $p$ is the farfield scattered pressure at distance $r=1 \times 10^{5} \mathrm{~m}$ 


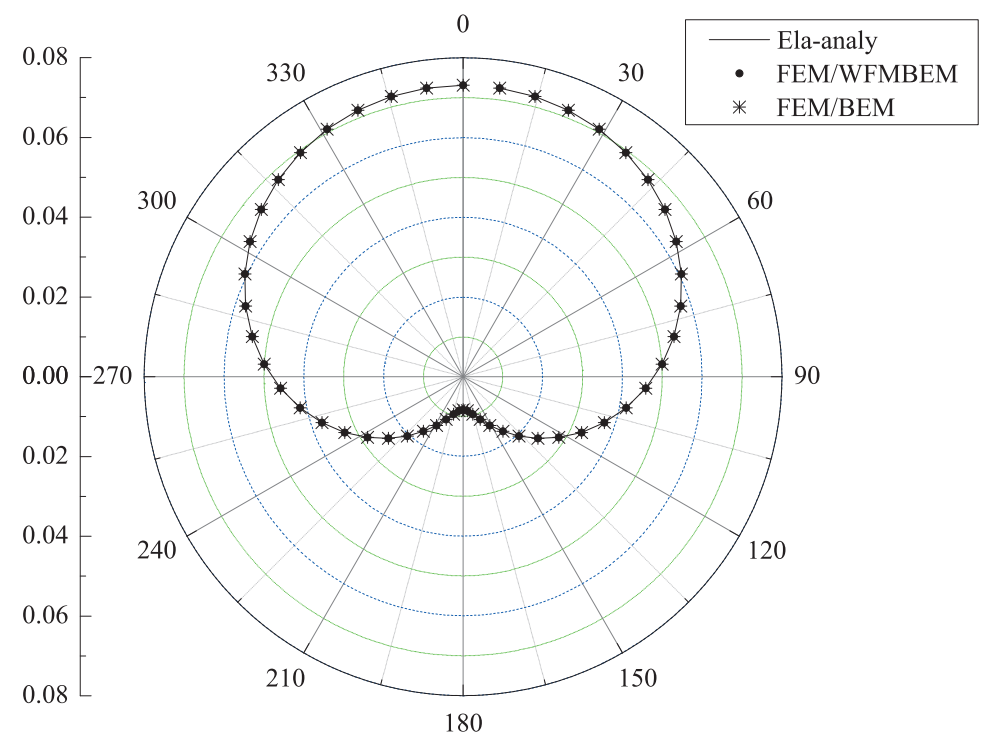

Figure 2: Normalized farfield pressure scattered in the $\mathbf{x y}$ plane.

from the origin point and $p_{o}$ is the magnitude of the incident pressure. This figure denotes that the numerical solutions based on the proposed algorithm in this paper agree with the analytical solutions well. Figure 3 shows that the Normalized

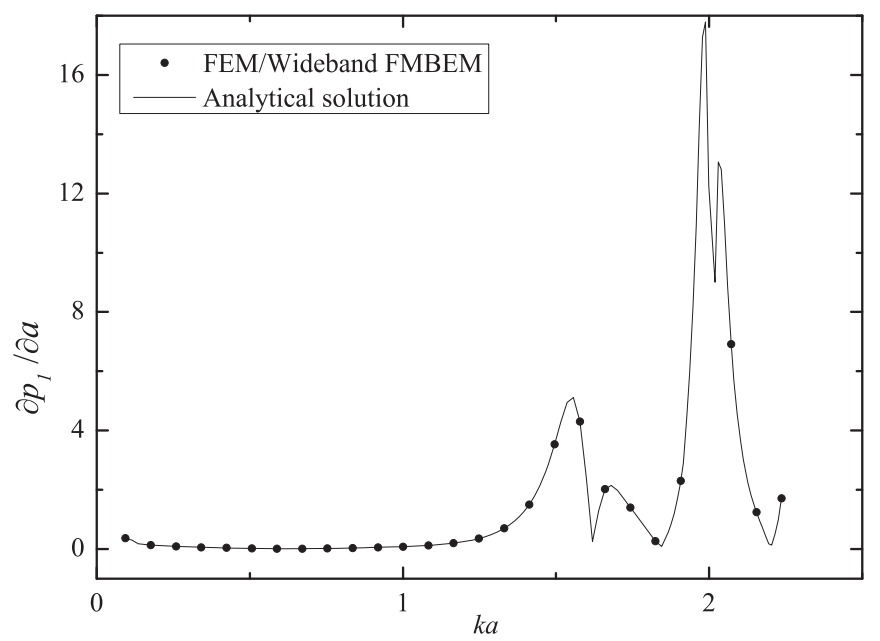

Figure 3: Normalized farfield pressure sensitivity values in the positive $\mathbf{x}$ axis with different $k a$. 


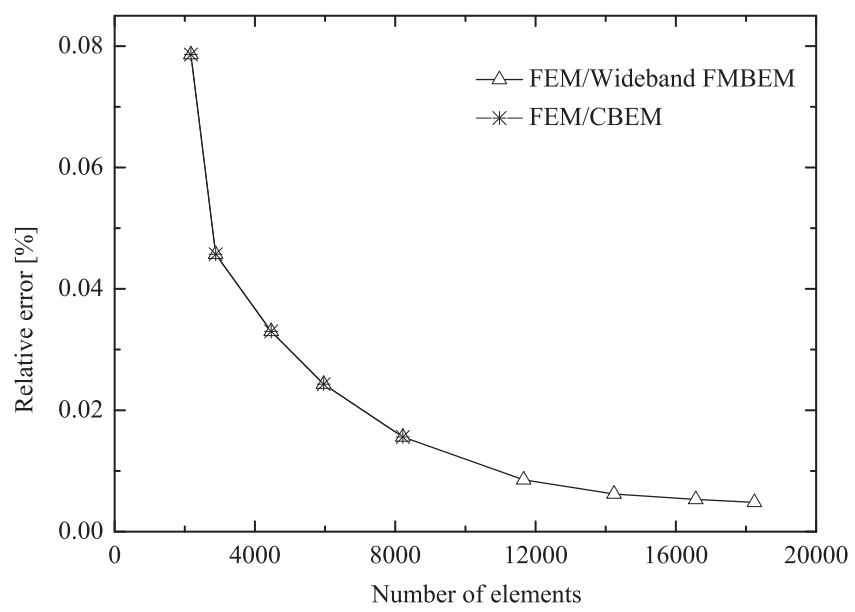

Figure 4: Relative error of the normalized farfield pressure sensitivity in the positive $\mathbf{x}$ axis with $k a=1.0$.

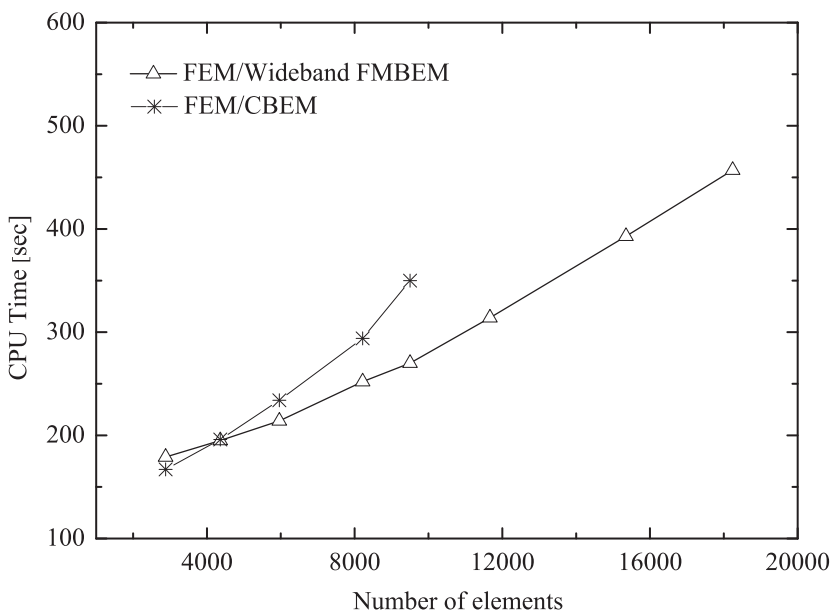

Figure 5: CPU time used to calculate the farfield pressure with $k a=1.0$.

farfield scattered pressure sensitivity values in the positive $\mathbf{x}$ axis obtained by using FEM/Wideband FMBEM algorithm agree well with analytical solutions and it implicates the accuracy of the presented algorithm, where the design variable is chosen as the radius $a$ of the spherical shell structure. The relative error is defined as

$$
\text { error }=\frac{\left|p_{\text {numer }}-p_{\text {analy }}\right|}{\left|p_{\text {analy }}\right|}
$$


where the $p_{\text {numer }}$ denotes the numerical solution and the $p_{\text {analy }}$ denotes the analytical solution. Actually, in the numerical evaluation of these boundary integral equations, truncation and numerical integration errors are the main errors. By observing Fig. 4, it can be found that the solution converges well when refining the boundary mesh and it implicates the accuracy of the presented algorithm. The CPU time used to calculate the farfield acoustic pressure values in the positive $\mathbf{x}$ axis is plotted in Fig. 5, which demonstrates the high efficiency of FEM/Wideband FMBEM algorithm for three dimensional fluid-structure interaction problems.

\section{Conclusions}

A coupling algorithm based on FEM and wideband FMBEM is presented for the simulation of fluid-structure interaction and structural acoustic sensitivity analysis using the direct differentiation method. The FEM was used to model the structural parts of the problem. To avoid the need to mesh the fluid domain, the wideband FMBEM formed by combining the original FMBEM and the diagonal form FMBEM is used to accelerate the matrix-vector products in the boundary element analysis. The presented algorithm makes it possible to predict the effects of arbitrarily shaped vibrating structures on the sound field numerically.

However, the iterative solution of the system of linear equation based on GMRES method is often the most time-consuming part of the simulation for modeling fluid-structure interaction problems numerically by using the coupling FEM/wideband FMBEM algorithm. The development of a more suitable preconditioner is required and this problem is now being addressed in an ongoing research project. Future work also includes applying the acoustic design sensitivity analysis to shape optimizations and extending the method to three dimensional practical problems.

\section{Acknowledgements}

Financial supports from the National Natural Science Foundation of China (NSFC) under Grant no. 11172291, China Postdoctoral Science Foundation under Grant no. 2012M510162 and the Fundamental Research Funds for the Central Universities (KB2090050024) are acknowledged.

\section{References}

[1] Everstine, G.C. and Henderson, F.M., Coupled finite element/boundary element approach for fluid-structure interaction. Journal of the Acoustic Society of America, 87(5), pp. 1938-1947, 1990.

[2] Fritze, D., Marburg, S. and Hardtke, H.J., FEM-BEM-coupling and structuralacoustic sensitivity analysis for shell geometries. Computers and Structures, 83(2-3), pp. 143-154, 2005. 
[3] Rokhlin, V., Diagonal forms of translation operators for the Helmholtz equation in three dimensions. Appl Comput Harmon Anal, 1, pp. 82-93, 1993.

[4] Cheng, H. and et al. A wideband fast multipole method for the Helmholtz equation in three dimensions. J Comput Phys, 216, pp. 300-325, 2006.

[5] Zheng, C.J., Matsumoto, T., Takahashi, T. and Chen, H.B., A wideband fast multipole boundary element method for three dimensional acoustic shape sensitivity analysis based on direct differentiation method. Engineering Analysis with Boundary Elements, 36, pp. 361-371, 2012.

[6] Chen, L.L., Zheng, C.J. and Chen, H.B., A wideband FMBEM for 2D acoustic design sensitivity analysis based on direct differentiation method. Computational Mechanics, 52, pp. 631-648, 2013.

[7] Coifman, R., Rokhlin, V. and Wandzura, S., The fast multipole method for the wave equation: A pedestrian prescription. IEEE Antennas Propag, 35, pp. 7-12, 1993.

[8] Junger, M.C. and Feit, D., (eds.) Structures and Their Interaction. MIT Press, 1985. 